Scottish border), a geographically distinct area with a stable population of three million, and approximately 35000 deliveries per year.

Participants: 13738 individuals with at least one congenital anomaly (classified according to the European Surveillance of Congenital Anomalies, EUROCAT, guidelines) born between 01 January 1985 and 31 December 2003, of whom 10951 were live born and 10837 (99\%) were traced for their survival status on 28 January 2008.

Main Outcome Measures: Congenital anomaly group and subtype specific survival rates at one week, four weeks, one year, five years, 10 years, 15 years and 20 years. Change in survival, and the number of terminations of pregnancy for fetal anomaly, with time.

Results: Survival among children with at least one congenital anomaly was $88.8 \%$ (95\% CI 88.2 to 89.4$)$ and 85.5 (95\% CI 84.8 to $86.3)$ at one year and 20 years respectively. Twenty year survival among the most common anomaly groups was $89.5 \%$ (95\% CI 88.4 to 90.6 ) for cardiovascular, $79.1 \%$ (95\% CI 76.7 to 81.3 ) for chromosomal, $93.2 \%$ (95\% CI 91.6 to 94.5$)$ for urinary, $83.1 \%(95 \%$ CI 79.6 to 86.0 ) for digestive system, $97.7 \%$ (95\% CI 96.1 to 98.7 ) for orofacial clefts, and $66.2 \%$ (95\% CI 61.5 to 70.4) for nervous system anomalies. Survival varied considerably between subtypes, even within the same anomaly group. The proportion of terminations for fetal anomaly increased throughout the study period, and, together with year of birth, was an independent predictor of survival.

Conclusions: This study presents robust estimates of survival, uniquely to age 20 years, for a range of congenital anomaly groups and subtypes, some of which have been infrequently reported previously. This information will be valuable for families, genetic councillors and other health care professionals when a congenital anomaly is detected, and will assist in planning for the future care needs of affected individuals.

\section{MEASURING LOCOMOTOR DISABILITY: A NEW SCORING MECHANISM FOR SOME FAMILIAR ITEMS}

S Muller, E Thomas, G Peat. Arthritis Research Campaign National Primary Care Centre, Primary Care Sciences, Keele University, Keele, Staffordshire, UK

\section{doi:10.1136/jech.2009.0967270}

Background: Locomotor disability is a critical measure for studying functional decline in later life and has featured in several major epidemiological studies. Yet measurement has mostly been confined to single items or ordinal scales rather than truly unidimensional measures with interval properties, thereby limiting the detection and interpretation of change in locomotor disability over time. Our aim was to combine items from the well-known SF-36 Physical Functioning Scale (PF-10) into a single score using Rasch analysis and test the properties of this new measure.

Methods: A health survey was sent to a random sample of 500 adults aged 50 years and over registered with a single general practice in North Staffordshire. The questionnaire contained the PF10 , from which five items (two relating to stair climbing and three to walking) were selected. Response dependency exists between the items, and so they were formed into two "super-items" (stairclimbing and walking). These items were assessed for their fit to the Rasch measurement model in terms of threshold ordering, unidimensionality, item and person fit, invariance along the latent trait and differential item functioning (DIF) by age-group (50-65, $66+$ years) and gender. Rasch analysis was carried out in RUMM2020. The score was confirmed in three external datasets and tests carried out for its repeatability and validity.

Results: 372 people responded to the health survey and of these, 368 responded to at least one of the five PF-10 items. Both superitems displayed disordered thresholds that were corrected by combining adjacent response categories. There was no evidence against unidimensionality, and item and person fit was good. There was no evidence against invariance along the latent trait and no signs of DIF by either age-group or gender. The items from the external datasets also met Rasch model specifications and the score was shown to be repeatable and valid.

Conclusions: Applications of measurement scales with confirmed interval level properties are advantageous for epidemiological studies using repeated measures over time. We have developed and externally validated a scoring method for deriving a brief selfreported locomotor disability scale with Rasch properties from commonly gathered items. This should allow the interval-level measurement of locomotor disability in general populations and so improve the quality of longitudinal studies in this area. The use of items from the PF-10 means that this improvement in quality is at no extra burden to the respondent or researcher, as in many cases, data can be analysed retrospectively.

\section{PHYSICAL CAPABILITY AND SUBSEQUENT MORTALITY: A SYSTEMATIC REVIEW AND META-ANALYSIS OF PUBLISHED DATA}

R Cooper, D Kuh, R Hardy, the FALCon study team. MRC Unit for Lifelong Health and Ageing, Department of Epidemiology and Public Health, Royal Free and University College Medical School, London, UK

doi:10.1136/jech.2009.096727p

Background: Evidence suggests that measures of physical capability may be predictive of subsequent disability, health and mortality, but the results from existing published studies have not been synthesised.

Objectives: To review published literature examining the associations between physical capability and subsequent all-cause mortality.

Design: Systematic review and meta-analysis.

Sources: Medline (from 1950 to 2008/05), EMBASE (from 1980 to 2008/02) and manual searches of the reference lists of eligible papers.

Included Studies: Any study examining the association between at least one of the specified measures of physical capability (grip strength, chair rises, standing balance, walking speed/time) and mortality in a population who were healthy and communitydwelling at baseline.

Results: Of 2240 unique records screened, 20 papers met the inclusion criteria of the review. An additional 3 papers were identified from reference lists. Within the 23 papers, there were 17 published analyses of grip strength, 9 of walking time/speed, 2 of standing balance and 1 of chair rises. The mean age of participants ranged from 36 to 80 y but the majority of study populations were aged $60 \mathrm{y}$ and above. Of the papers investigating the association between grip strength and mortality, 6 presented Hazard Ratios for a unit change in grip strength with basic adjustments (total $\mathrm{n}=10171$, deaths $=4267$ ). The pooled Hazard Ratio for mortality per $5 \mathrm{~kg}$ increase in grip strength, from a random effects meta-analysis, was 0.90 (95\% CI 0.87 to 0.93). Heterogeneity between studies $\left(\mathrm{I}^{2}=65.2 \%\right)$ was largely explained by a single study which had weaker results. The pooled HR when this study was removed was 0.89 (0.86 to 0.92$)\left(\mathrm{I}^{2}=25.7 \%\right)$. Other papers, where comparisons between categories of grip strength had been performed, also generally found weaker grip strength to be associated with higher rates of mortality but these analyses were too heterogeneous to be combined using meta-analysis. Due to differences in the ways in which walking speed had been measured and analysed it was not possible to perform meta-analyses of these data, but the results from all 9 studies showed that slower walking speed/longer walking time was significantly associated with increased mortality risk.

Conclusions: This review demonstrates that grip strength and walking time/speed are useful markers of subsequent mortality risk in community-dwelling populations. The underlying explanations 